\title{
Physical activity in the classroom to prevent childhood obesity: A pilot study in Santiago, Chile - CORRIGENDUM
}

In the above mentioned article ${ }^{(1)}$ the authors were given in the wrong order. The correct order should have been

Pilar Arnaiz, Johana Soto-Sánchez, Juana Saavedra, Angélica Domínguez, Jaime Rozowski, Laura Iriarte, Jennifer Cantwell Wood and Francisco Mardones.

Additionally, Johana Soto-Sánchez's affiliation was given incorrectly, it should have read:

${ }^{3}$ Department of Physical Education, Faculty of Physical and Sport Sciences, Universidad de Playa Ancha, Chile.

\section{Reference}

1. Mardones F, Arnaiz P, Soto-Sánchez J, et al. (2017). Physical activity in the classroom to prevent childhood obesity: A pilot study in Santiago, Chile. Journal of Nutritional Science, 6. doi:10.1017/jns.2017.14. 